\title{
Antibody titers by MAT and IHA in phage lysate immunized brucellosis affected cows
}

\author{
Raj S and Saxena HM* \\ Department of Veterinary Microbiology, College of Veterinary Science, Guru Angad Dev Veterinary and Animal Sciences University, Ludhiana, India
}

\begin{abstract}
Brucellosis, caused by Brucella organisms, is an important zoonotic disease causing huge economic losses to the livestock industry worldwide. The affected animals remain carrier throughout their lives. Currently there is no satisfactory and cost-effective treatment for bovine Brucellosis. Antibiotic resistance has also been reported in Brucella organisms. We have carried out immunization of bovine Brucellosis affected cows by phage lysates of Brucella abortus attenuated strains RB51 and S19. A single dose of $2 \mathrm{ml}$ of the S19 lysate or the cocktail lysate, comprising lysates of both RB51 and S19, administered subcutaneously to naturally Brucellosis affected adult cattle induced strong antibody response in cattle. Results of both, MAT and IHA, indicated that in cattle treated with the S19 lysate or cocktail lysate, the differences between mean titres of $0 \mathrm{D}$ and $90 \mathrm{D}$ were very significant $(\mathrm{P}<0.01)$. However, in case of RB51 lysate treated cattle and in Brucellosis affected untreated cattle, the variation in the titers at various intervals was not significant.
\end{abstract}

\section{Introduction}

Brucellosis is endemic in India and is prevalent in all parts of the country. It causes heavy economic loss to the animal industry through delayed conception, late-term abortions and retention of placenta and temporary or permanent infertility [1] in females and orchitis and epididymitis in males, with excretion of organisms in semen, uterine discharges, and in milk [2].

Numerous outer and inner membrane, cytoplasmic, and periplasmic protein antigens of Brucella have been characterized, yet the antigen that dominates the antibody response is Lipopolysaccharide (LPS). Some antigens are recognized by the immune system during infection and are potentially useful in diagnostic tests. The L7/L12 ribosomal proteins are important in stimulating cell-mediated responses [3].

The role of humoral immunity against intracellular bacterial infections is limited and may not be protective. Antibody mediated opsonization by immunoglobulins (IgM, IgG1, IgG2a and IgG3) enhances phagocytic uptake of bacteria, limiting the level of initial infection with Brucella but has little effect on intracellular course of infection [4].

We explored the immunotherapeutic potential of phage lysates of S19 and RB51 in Brucellosis in adult cattle and employed MAT and IHA to monitor the effect of lysate therapy on the antibody titers in cattle.

\section{Materials and methods \\ Brucella abortus vaccine strains}

Live organisms from the attenuated vaccines Brucella abortus strain 19 and strain RB51 (Indian Immunologicals, Hyderabad) were used for making phage lysates for immunotherapy. The bacterial cultures were maintained on Brucella agar plates, Trypticase Soy Agar (TSA) and Farrell's medium (OIE 2009) and slants by serial subculturing in Brucella selective broth on every fortnight and storing the cultures at $4^{\circ} \mathrm{C}$.

\section{Brucellaphage}

A broad acting phage lytic to Brucella organisms isolated in our laboratory [5] was used for lysing Brucella abortus strains 19 and RB51 for making lysates. Phage as a crude, concentrated suspension prepared in SM diluent, was first revived by agar overlay technique as per the procedure reported earlier [6]. The phage preparation obtained as mentioned above was amplified to $250 \mathrm{ml}$ master lot using the liquid culture method [7].

\section{Generation of phage lysates of Brucella}

The methodology for generation of phage lysate preparations against Brucella abortus using Brucellaphage has been reported earlier [8]. The total viable count (TVC) of 24-48 hr Brucella selective broth culture of the strains $\mathrm{S} 19$ and $\mathrm{RB} 51$ incubated at $37^{\circ} \mathrm{C}$ was adjusted to $2 \times 10^{8} \mathrm{cfu} / \mathrm{ml}$. Opacity of broth was adjusted to Mac Farland's tube no 3 for the purpose of fetching appropriate antigenic biomass. Phage was added as per optimized MOI and TVC of the respective indicator strains (with phage bacteria ratio of 1: 50) and the mixture was further incubated for 6-7 hours at $37^{\circ} \mathrm{C}$ for complete lysis and clearance of turbidity. $100 \mathrm{ml}$ each of phage lysate was prepared against both the strains (Brucella abortus S 19 and RB 51).

The phage lysate cocktail was then passed through a $0.1 \mu \mathrm{m}$ filter (Pall Life Science) to separate out the phage from the lysate and the filtrate was stored in sterilized vials at $4{ }^{\circ} \mathrm{C}$. Sterile aluminium hydroxide gel suspension in saline in ratio of 1:10 was added to the preparation. Total protein content of the phage lysate, as determined by Nanodrop

${ }^{\star}$ Correspondence to: Hari Mohan Saxena, Department of Veterinary Microbiology, College of Veterinary Science, Guru Angad Dev Veterinary and Animal Sciences University, Ludhiana, India, E-mail: drhmsaxena@gmail.com

Key words: antibody titer, brucella, brucellosis, brucellaphage, phage lysate, bacteriophage, microtiter plate agglutination test, indirect haemagglutination test

Received: December 28, 2018; Accepted: January 16, 2019; Published: January 18,2019 
spectrophotometer, was $0.58 \mathrm{mg} / \mathrm{ml}$ for S19 lysate and $0.64 \mathrm{mg} / \mathrm{ml}$ for RB 51 lysate. The phage lysate cocktail (combination of Brucella abortus S19 and RB51 lysates) was prepared by addition of equal quantity of S19 phage lysate and RB51 lysate. Sterilized 1\% Aluminium hydroxide gel suspension in saline was mixed aseptically with the test preparations in ratio of 1:10 (final Aluminium concentration $0.1 \%$ ) and incubated at $37^{\circ} \mathrm{C}$ for 24 hours and then stored at $4^{\circ} \mathrm{C}$. The phage lysates were then subjected to sterility and safety tests.

\section{Sterility testing of lysates}

A loopful of the lysate was suspended in $5 \mathrm{ml} \mathrm{BHI} \mathrm{and} \mathrm{BSM} \mathrm{broth}$ as well as streaked on BHI and BSM, Trypticase Soy Agar followed by incubation at $37^{\circ} \mathrm{C}$. The broth and plates were examined up to 48 hours for any microbial growth.

\section{Compliance with animal ethics}

All the experimental protocols performed on mice and cattle were approved by the Institutional Animal Ethics Committee (IAEC) of GADVASU, Ludhiana. Animals were kept in approved facilities as per the CPCSE guidelines.

\section{Safety test of the phage lysates in mice}

Safety test of all the preparations of lysates was conducted in mice as recommended in the Indian Pharmacopoeia 2010 before commencement of immunization of cattle. Three groups of three adult healthy mice, each were injected with $0.2 \mathrm{ml}$ volume of lysate (S19 or RB51 or cocktail of both) by subcutaneous route. One group of three adult healthy mice was left untreated as control. The mice were observed for any untoward reaction or mortality up to $7^{\text {th }}$ day of inoculation.

\section{Immunotherapy trials in cattle}

Trials of phage lysate therapy were carried out on 21 naturally Brucellosis affected adult cows. The Brucellosis positive adult cows were divided into four groups:

Group I animals ( $\mathrm{n}=6)$ were immunized with Brucella abortus S19 lysate only, Group II animals $(\mathrm{n}=5)$ were immunized with Brucella abortus RB51 lysate only, Group III animals $(n=5)$ were immunized with cocktail of S19 and RB51 lysates. A dose of $2 \mathrm{ml}$ lysate was administered through subcutaneous route whereas Group IV $(n=5)$ served as a control and received no immunization.

Blood samples from cattle were collected through jugular vein at 0 day and at 30,60, 75 and 90 days post treatment for studying the immune response of the animals specific to the Brucella organisms. Sera were separated from blood and stored at $-20^{\circ} \mathrm{C}$ and $-80^{\circ} \mathrm{C}$, respectively till further use.

\section{Rose bengal plate test (RBPT)}

Equal volumes $(25 \mu \mathrm{l}$ each) of RBPT colored antigen (Punjab Veterinary Vaccine Institute, Ludhiana) and test serum were mixed on a clean glass slide [9]. The slide was observed till $3 \mathrm{~min}$. for formation of clumps. Formation of clumps indicated a positive reaction while the absence of clear clumps was considered as a negative reaction.

\section{Microtiter plate agglutination test (MAT)} (1971)

MAT was performed as per the method of Williams and Whittemore

a. Serum samples were serially two-fold diluted in phosphate buffer saline to make final volume of $100 \mu \mathrm{l}$ in a 96 well $\mathrm{U}$ bottom microtiter plate (Tarsons). b. Equal volume of $100 \mu \mathrm{l}$ of Plain Brucella abortus antigen (Punjab Veterinary Vaccine Institute, Ludhiana) was added to each well.

c. Negative control well containing $100 \mu$ l Phosphate buffer saline and $100 \mu$ antigen was also marked.

d. The plate was sealed and mixed gently for 20 seconds and incubated at $37^{\circ} \mathrm{C}$ for $24 \mathrm{hr}$ followed by incubation at $4^{\circ} \mathrm{C}$ for 1 hour.

Titers $\left(\log _{10}\right.$ values) were expressed as reciprocal of the highest dilution of sera showing 50 percent agglutination. Matt formation was considered as a positive reaction while button formation was considered as a negative reaction.

\section{Indirect haemagglutination test (IHA)}

The Method of Sawada et al (1982) was followed with slight modifications [10-12].

\section{Fixation of sheep red blood cells (sRBCs)}

Sheep blood was collected aseptically into Alsever's solution (1:1) by jugular vein puncture and kept at $4^{\circ} \mathrm{C}$ for 7 days before further processing. Blood was centrifuged at $1500-2000 \mathrm{rpm}$ for $10 \mathrm{~min}$ to settle the RBCs. The packed RBCs were washed three times with 5-6 volumes of chilled NSS by centrifugation. The $10 \%$ suspension of RBCs prepared in chilled NSS was stored at $4^{\circ} \mathrm{C}$.

\section{Fixation of RBCs and treatment of G-sRBCs with tannic acid}

One percent solution of glutaraldehyde was prepared in NSS and stored at $4^{\circ} \mathrm{C}$ for $30 \mathrm{~min}$ with intermittent gentle stirring.

The sensitized sRBCs were packed by centrifugation at 1500-2000 $\mathrm{rpm}$ for $10 \mathrm{~min}$ at room temperature followed by three washes in normal saline for removal of glutaraldehyde. This was resuspended in the same buffer containing 0.1 percent sodium azide to yield a 10 percent suspension of sRBCs. The glutaraldehyde fixed sRBCs (G-sRBCs) were stored at $4^{\circ} \mathrm{C}$.

Ten percent suspension of G-sRBCs was mixed with equal volume of Phosphate Buffered Saline containing 0.005 percent tannic acid $(\mathrm{w} / \mathrm{v})$, and incubated at $37^{\circ} \mathrm{C}$ with occasional shaking. The tanned G-sRBCs (TG-sRBCs) were pelleted by centrifugation at $650 \mathrm{x} g$ for 10 min at room temperature and washed three times with PBS to yield a 10 percent suspension.

\section{Preparation of antigen}

The antigen prepared as described earlier was heated at $56^{\circ} \mathrm{C}$ for 30 min in a water bath with frequent shaking. Heat treated suspension was centrifuged at $8000 \mathrm{rpm}$ for $15 \mathrm{~min}$ at $4^{\circ} \mathrm{C}$. The clear supernatant was separated and stored at $-20^{\circ} \mathrm{C}$.

\section{Sensitization of TG-sRBCs with antigen}

One volume of packed RBCs and 15 volumes of the antigen were mixed and incubated for $1-2 \mathrm{hr}$ at $37^{\circ} \mathrm{C}$ in a water bath with frequent shaking. The sensitized cells prepared were washed three times with NSS by centrifugation at $2500 \mathrm{rpm}$ for $10 \mathrm{~min}$. After the final wash, the packed cells were resuspended in chilled NSS to obtain 1 percent suspension.

\section{Adsorption of serum samples}

To remove the heterophile antibodies, all the serum samples were adsorbed with packed sRBCs ( 1 volume) for 2 hours at $37^{\circ} \mathrm{C}$ with periodic shaking before the test proper. The RBCs were removed by 
centrifugation. The suspension was centrifuged at $600 \mathrm{xg}$ for $15 \mathrm{~min}$ at $4^{\circ} \mathrm{C}$ in a refrigerated centrifuge. The suspension was collected and used for the test.

\section{Test Protocol for IHA}

Phosphate buffered saline $(160 \mu \mathrm{l})$ and inactivated adsorbed serum

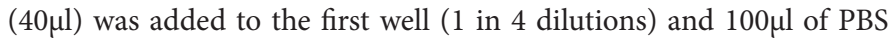
was added to all the wells. The plates were shaken and left at room temperature for $2 \mathrm{hr}$. Coarse agglutination of RBCs (matt formation) indicated a positive result and formation of small button of deposited cells was considered as a negative result.

\section{Controls}

Three controls were included in the test:

Antigen control: $100 \mu \mathrm{l}$ of sensitized and adsorbed RBCs. RBCs.

RBC control: $100 \mu \mathrm{l}$ of 1:4 dilution of serum and $100 \mu$ l of sensitized

Serum control: $100 \mu$ of untreated erythrocytes and $100 \mu \mathrm{l}$ of test serum.

Statistical analysis: Data pertaining to serum antibody titers by MAT and IHA were statistically analyzed by ' $\mathrm{t}$ ' test and ANOVA.

\section{Results and discussion}

\section{Immunization of Brucellosis affected cattle with phage lysate}

Brucellosis affected cattle were treated with Brucella abortus strain 19 and RB51 phage lysate vaccines. Pre ( 0 day) and post treatment serum samples were collected at $0,30,60,75$ and 90 days and stored at $-20^{\circ} \mathrm{C}$ and $-80^{\circ} \mathrm{C}$ respectively until used. All the serum samples from the infected animals under study showed positive reaction with RBPT during the entire period since the anitbody developed against Brucella organisms gives the agglutination reaction with the Rose Bengalcoloured antigen.

\section{Titers of anti-Brucella antibodies in cattle treated with lysates}

The present study was undertaken to explore the therapeutic potential of phage lysate of Brucella abortus vaccine strains S19 and RB 51. Brucellaphage isolated in our laboratory was used to prepare the phage lysates.

Cattle naturally infected with Brucellosis were treated with either S19 lysate or RB51 lysate or a combination of both (cocktail lysate). The therapeutic vaccines (phage lysates) were injected in the experimental animals at a dose of $2 \mathrm{ml}$ subcutaneously. The controls were left untreated. Antibody titers in sera of animals were determined by MAT and IHA at various intervals $(0,30,60,75$ and 90 days). All the serum samples from the infected animals under study showed positive reaction with RBPT. The antibody titers showed a classical pattern of gradual rise from prevaccination levels to achievement of peaks followed by plateau and then gradual decline over a period of time.

\section{Titers by MAT}

The antibody titers as recorded by MAT are shown in Tables 1-4 and Figures 1 and 2. In case of S19 lysate immunized animals, the mean titers varied from $2.856+0.58$ on 0 day to $3.257+0.41$ on 30 day, 3.458 +0.48 on 60 day, $3.709+0.42$ on 75 day and $4.060+0.29$ on 90 , day respectively. The differences between mean values of $0 \mathrm{D}$ and $75 \mathrm{D}$ and between 30D and 90D were significant $(\mathrm{P}<0.05)$ and between $0 \mathrm{D}$ and
90D was very significant $(\mathrm{P}<0.01)$. This could be expected because Brucella abortus strain 19 is reported to induce antibody response but does not affect the cell mediated immune response in the host to a large extent.

In case of RB51 lysate immunized animals, the mean titers ranged from $2.806+0.60$ on 0 day to $3.297+0.34$ on 30 day, $3.046+0.39$ on 60 day, $2.926+0.34$ on 75 day, and $2.986+0.34$ on 90 day, respectively. The differences among the mean values were non-significant at different intervals. This response could be expected because Brucella abortus RB51 strain is known to induce cellular immune response and does not induce detectable humoral immunity.

In cattle treated with the cocktail lysate, the mean titers ranged from $2.745+0.39$ on 0 day to $3.287+0.34$ on 30 day, $3.287+0.45$ on 60 day, $3.889+0.34$ on 75 day and $4.070+0.25$ on 90 day, respectively. The increase in the titers from 30 day to 60 day and between 30 day to 90 day was significant $(\mathrm{P}<0.05)$. Similarly, the increase from 0 day to 75 day and 0 day to 90 day was very significant $(\mathrm{P}<0.01)$ Tables 5-8.

In case of Brucellosis affected untreated (control) cattle, titers ranged from $3.046+0.49$ on 0 day to $2.986+0.45$ on 30 day, 2.986 +0.45 on 60 day, $3.167+0.39$ on 75 day and $2.806+0.30$ on 90 day, respectively. The variation in the titers at various intervals was not significant.

Table 1. Antibody titers by MAT in Brucellosis affected cattle treated with S19 lysate

\begin{tabular}{|c|c|c|c|c|c|}
\hline Animal no. & $\mathbf{0}$ D & $\mathbf{3 0} \mathbf{D}$ & $\mathbf{6 0} \mathbf{D}$ & $\mathbf{7 5} \mathbf{D}$ & $\mathbf{9 0} \mathbf{D}$ \\
\hline 1469 & 3.709 & 3.709 & 4.010 & 4.010 & 4.311 \\
\hline 1456 & 2.505 & 2.806 & 3.107 & 3.408 & 3.709 \\
\hline 1405 & 2.806 & 3.408 & 3.408 & 3.709 & 4.010 \\
\hline 1397 & 3.408 & 3.709 & 4.010 & 4.311 & 4.311 \\
\hline 1376 & 2.505 & 2.806 & 2.806 & 3.107 & 3.709 \\
\hline 1440 & 2.204 & 3.109 & 3.408 & 3.709 & 4.311 \\
\hline Mean $\pm \mathbf{S D}$ & $\mathbf{2 . 8 5 6} \pm \mathbf{0 . 5 8}$ & $\mathbf{3 . 2 5 7} \pm \mathbf{0 . 4 1}$ & $\mathbf{3 . 4 5 8} \pm \mathbf{0 . 4 8}$ & $\mathbf{3 . 7 0 9} \pm \mathbf{0 . 4 2}$ & $\mathbf{4 . 0 6 0} \pm \mathbf{0 . 2 9}$ \\
\hline
\end{tabular}

Table 2. Antibody titers by MAT in Brucellosis affected cattle treated with RB51 lysate

\begin{tabular}{|c|c|c|c|c|c|}
\hline Animal no. & $\mathbf{0 ~ D}$ & $\mathbf{3 0} \mathbf{D}$ & $\mathbf{6 0} \mathbf{D}$ & $\mathbf{7 5} \mathbf{D}$ & $\mathbf{9 0} \mathbf{D}$ \\
\hline 1479 & 2.505 & 3.408 & 3.408 & 3.107 & 3.408 \\
\hline 1444 & 2.204 & 2.806 & 2.505 & 2.505 & 2.806 \\
\hline 1495 & 3.709 & 3.709 & 3.408 & 3.408 & 3.107 \\
\hline 1281 & 2.505 & 3.107 & 2.806 & 2.806 & 2.505 \\
\hline 1385 & 3.107 & 3.408 & 3.107 & 2.806 & 3.107 \\
\hline Mean \pm SD & $\mathbf{2 . 8 0 6} \pm \mathbf{0 . 6 0}$ & $\mathbf{3 . 2 8 7} \pm \mathbf{0 . 3 4}$ & $\mathbf{3 . 0 4 6} \pm \mathbf{0 . 3 9}$ & $\mathbf{2 . 9 2 6} \pm \mathbf{0 . 3 4}$ & $\mathbf{2 . 9 8 6} \pm \mathbf{0 . 3 4}$ \\
\hline
\end{tabular}

Table 3. Antibody titers by MAT in Brucellosis affected cattle treated with cocktail lysate

\begin{tabular}{|c|c|c|c|c|c|}
\hline Animal no. & $\mathbf{0 ~ D}$ & $\mathbf{3 0} \mathbf{D}$ & $\mathbf{6 0} \mathbf{D}$ & $\mathbf{7 5} \mathbf{D}$ & $\mathbf{9 0} \mathbf{D}$ \\
\hline 1367 & 2.505 & 3.408 & 3.408 & 4.010 & 4.311 \\
\hline 1390 & 2.806 & 2.806 & 2.806 & 3.408 & 3.709 \\
\hline 1418 & 2.505 & 3.107 & 2.806 & 4.010 & 4.010 \\
\hline 1460 & 2.505 & 3.406 & 3.709 & 3.709 & 4.010 \\
\hline 1471 & 3.408 & 3.709 & 3.709 & 4.311 & 4.311 \\
\hline Mean \pm SD & $\mathbf{2 . 7 4 5} \pm \mathbf{0 . 3 9}$ & $\mathbf{3 . 2 8 7} \pm \mathbf{0 . 3 4}$ & $\mathbf{3 . 2 8 7} \pm \mathbf{0 . 4 5}$ & $\mathbf{3 . 8 8 9} \pm \mathbf{0 . 3 4}$ & $\mathbf{4 . 0 7 0} \pm \mathbf{0 . 2 5}$ \\
\hline
\end{tabular}

Table 4. Antibody titers by MAT in Brucellosis affected untreated cattle

\begin{tabular}{|c|c|c|c|c|c|}
\hline Animal no. & $\mathbf{0 ~ D}$ & $\mathbf{3 0} \mathbf{D}$ & $\mathbf{6 0} \mathbf{D}$ & $\mathbf{7 5} \mathbf{D}$ & $\mathbf{9 0} \mathbf{D}$ \\
\hline 1408 & 2.806 & 2.806 & 2.806 & 3.408 & 3.107 \\
\hline 1493 & 2.505 & 2.204 & 2.505 & 2.806 & 2.505 \\
\hline 1351 & 3.408 & 3.408 & 3.107 & 2.806 & 2.505 \\
\hline 1319 & 3.709 & 3.709 & 3.709 & 3.709 & 3.107 \\
\hline 1382 & 2.806 & 2.806 & 2.806 & 3.107 & 2.806 \\
\hline Mean \pm SD & $\mathbf{3 . 0 4 6} \pm \mathbf{0 . 4 9}$ & $\mathbf{2 . 9 8 6} \pm \mathbf{0 . 5 8}$ & $\mathbf{2 . 9 8 6} \pm \mathbf{0 . 4 5}$ & $\mathbf{3 . 1 6 7} \pm \mathbf{0 . 3 9}$ & $\mathbf{2 . 8 0 6} \pm \mathbf{0 . 3 0}$ \\
\hline
\end{tabular}


It has been reported by Araya et al (1989) earlier that the live Brucella S19 induced immunity involves protective humoral responses against lipopolysaccharide $\mathrm{O}$ chain and a variety of proteins.

In case of $\mathrm{S} 19$ phage lysate immunized cows, the antibody titres by IHA ranged from $2.605+0.52$ on 0 day to $2.956+0.45$ on 30 day, $3.508+0.45$ on 60 day, $3.760+0.35$ on 75 day and $4.160+0.25$ on 90 day, respectively. The increase in titers from 0 to 60 day and 30 to 75 day was significant $(\mathrm{P}<0.05)$. The increase in the titers from 0 to 60 day, 75 day and 90 day and between 30 and 90 day was found to be very significant $(\mathrm{P}<0.01)$.



Figure 1. Antibody titters by MAT in lysate immunized cattle. (Titers by Indirect Haemagglutination Assay)

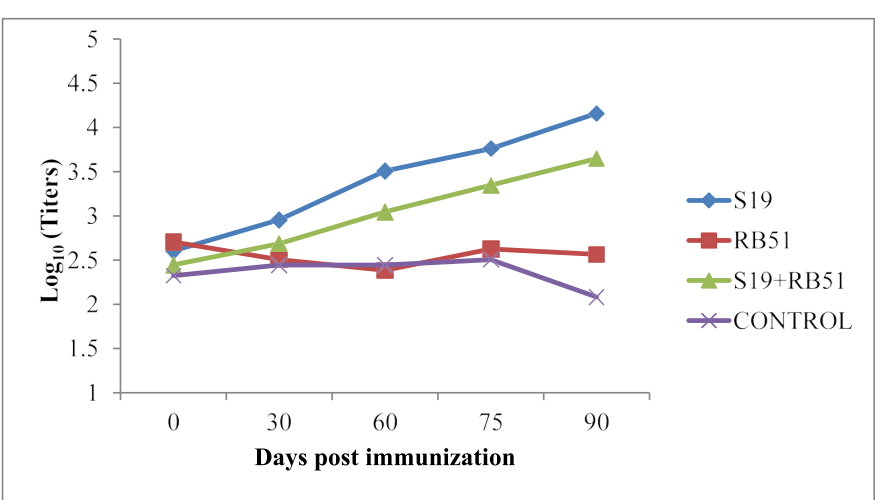

Figure 2. Antibody titers by IHA in lysate immunized cattle

Table 5. Antibody titers by IHA in Brucellosis affected cattle treated with S19 lysate

\begin{tabular}{|c|c|c|c|c|c|}
\hline Animal no. & $\mathbf{0 ~ D}$ & $\mathbf{3 0} \mathbf{D}$ & $\mathbf{6 0} \mathbf{D}$ & $\mathbf{7 5} \mathbf{D}$ & $\mathbf{9 0} \mathbf{D}$ \\
\hline 1469 & 3.408 & 3.709 & 4.010 & 4.013 & 4.311 \\
\hline 1456 & 2.204 & 2.505 & 2.806 & 3.107 & 3.709 \\
\hline 1405 & 2.806 & 3.107 & 3.709 & 4.013 & 4.311 \\
\hline 1397 & 2.806 & 2.806 & 3.709 & 4.013 & 4.311 \\
\hline 1376 & 2.505 & 3.107 & 3.709 & 3.709 & 4.010 \\
\hline 1440 & 1.903 & 2.505 & 3.109 & 3.709 & 4.311 \\
\hline Mean \pm SD & $\mathbf{2 . 6 0 5} \pm \mathbf{0 . 5 2}$ & $\mathbf{2 . 9 5 6} \pm \mathbf{0 . 4 5}$ & $\mathbf{3 . 5 0 8} \pm \mathbf{0 . 4 5}$ & $\mathbf{3 . 7 6 0} \pm \mathbf{0 . 3 5}$ & $\mathbf{4 . 1 6 0 \pm 0 . 2 5}$ \\
\hline
\end{tabular}

Table 6. Antibody titers by IHA in Brucellosis affected cattle treated with RB51 lysate

\begin{tabular}{|c|c|c|c|c|c|}
\hline Animal no. & $\mathbf{0 ~ D}$ & $\mathbf{3 0}$ D & $\mathbf{6 0}$ D & $\mathbf{7 5}$ D & 90 D \\
\hline 1479 & 2.404 & 2.204 & 1.903 & 2.204 & 2.505 \\
\hline 1444 & 2.204 & 1.903 & 1.903 & 2.505 & 2.505 \\
\hline 1495 & 3.408 & 3.107 & 3.107 & 3.107 & 2.806 \\
\hline 1281 & 2.404 & 2.204 & 2.204 & 2.505 & 2.204 \\
\hline 1385 & 3.107 & 3.107 & 2.806 & 2.806 & 2.806 \\
\hline Mean \pm SD & $\mathbf{2 . 7 0 5} \pm \mathbf{0 . 5 2}$ & $\mathbf{2 . 5 0 5} \pm \mathbf{0 . 5 6}$ & $\mathbf{2 . 3 8 4} \pm \mathbf{0 . 5 4}$ & $\mathbf{2 . 6 2 5} \pm \mathbf{0 . 3 4}$ & $\mathbf{2 . 5 6 5} \pm \mathbf{0 . 2 5}$ \\
\hline
\end{tabular}

Table 7. Antibody titers by IHA in Brucellosis affected cattle treated with cocktail lysate

\begin{tabular}{|c|c|c|c|c|c|}
\hline Animal no. & $\mathbf{0 ~ D}$ & $\mathbf{3 0} \mathbf{D}$ & $\mathbf{6 0} \mathbf{D}$ & $\mathbf{7 5} \mathbf{D}$ & $\mathbf{9 0} \mathbf{D}$ \\
\hline 1367 & 2.205 & 2.806 & 2.806 & 3.107 & 3.408 \\
\hline 1390 & 2.204 & 2.505 & 3.107 & 3.408 & 3.709 \\
\hline 1418 & 1.903 & 1.903 & 2.505 & 2.806 & 3.107 \\
\hline 1460 & 2.505 & 2.505 & 3.107 & 3.408 & 3.709 \\
\hline 1471 & 3.408 & 3.709 & 3.709 & 4.010 & 4.311 \\
\hline Mean \pm SD & $\mathbf{2 . 4 4 5} \pm \mathbf{0 . 5 7}$ & $\mathbf{2 . 6 8 5} \pm \mathbf{0 . 6 5}$ & $\mathbf{3 . 0 4 6} \pm \mathbf{0 . 4 4}$ & $\mathbf{3 . 3 4 7} \pm \mathbf{0 . 4 4}$ & $\mathbf{3 . 6 4 8} \pm \mathbf{0 . 4 4}$ \\
\hline
\end{tabular}

Table 8. Antibody titers by IHA in Brucellosis affected untreated cattle

\begin{tabular}{|c|c|c|c|c|c|}
\hline Animal no. & $\mathbf{0 ~ D}$ & $\mathbf{3 0} \mathbf{D}$ & $\mathbf{6 0} \mathbf{D}$ & $\mathbf{7 5} \mathbf{D}$ & $\mathbf{9 0} \mathbf{D}$ \\
\hline 1408 & 1.903 & 2.204 & 2.505 & 2.806 & 2.505 \\
\hline 1493 & 2.204 & 2.505 & 1.903 & 2.204 & 1.903 \\
\hline 1351 & 3.107 & 2.806 & 3.107 & 2.806 & 2.204 \\
\hline 1319 & 1.903 & 2.505 & 1.903 & 2.204 & 1.603 \\
\hline 1382 & 2.505 & 2.204 & 2.806 & 2.505 & 2.204 \\
\hline Mean \pm SD & $\mathbf{2 . 3 2 4} \pm \mathbf{0 . 5 0}$ & $\mathbf{2 . 4 4 4} \pm \mathbf{0 . 2 5}$ & $\mathbf{2 . 4 4 4} \pm \mathbf{0 . 5 3}$ & $\mathbf{2 . 5 0 5} \pm \mathbf{0 . 3 0}$ & $\mathbf{2 . 0 8 3} \pm \mathbf{0 . 3 4}$ \\
\hline
\end{tabular}

This study has revealed that even the phage lysed Brucella abortus S19 organisms retain sufficient immunogenicity to induce high magnitude of antibody response, lasting for long periods.

In case of RB51 lysate immunized animals, the mean titers ranged from $2.705+0.52$ on 0 day to $2.505+0.56$ on 30 day $2.384+0.54$ on 60 day, $2.625+0.34$ on 75 day and $2.565+0.25$ on 90 day, respectively. The titers at various intervals did not vary significantly.

In case of animals treated with cocktail lysate vaccine, the titers ranged from $2.445+0.57$ on 0 day to $2.685+0.65$ on 30 day, 3.046 +0.44 on 60 day, $3.347+0.44$ on 75 day, and $3.648+0.44$ on 90 day respectively. The increase in titers from 0 to 90 day was significant $(\mathrm{P}<0.05)$.

In Brucellosis affected cattle which did not receive any treatment, the titers ranged from $2.324+0.5$ on 0 day to $2.444+0.25$ on 30 day, $2.444+0.53$ on 60 day, $2.505+0.30$ on 75 day and $2.083+0.34$ on 90 day respectively. The differences among the titers at various intervals were non-significant.

The results indicate that the antibody titers enhanced considerably by Brucella abortus S19 lysate, similar to the earlier reports with whole live S19 vaccine. Interestingly the cocktail lysate generated a robust antibody response which consistently increased very significantly over 3 months' period of observation.

\section{Conclusion}

Phage lysate of Brucella abortus attenuated strain S19 alone or in combination with lysate of Brucella abortus attenuated strain RB51 administered subcuteneously at a single dose of $2 \mathrm{ml}$ in naturally Brucellosis infected adult cows led to significant increase in antibody titers at various intervals as estimated by MAT and IHA and can be used for immunotherapy in adult cattle.

\section{Competing interest}

The authors declare that no competing interests exist.

\section{Author contributions}

Investigation \& Methodology: Sugandha Raj \& H M Saxena; Validation: Sugandha Raj;

Conceptualization, data curation, formal analysis, funding acquisition, project administration, resources, supervision, visualization, writing - original draft, review \& editing: H M Saxena. 


\section{Acknowledgement}

The study was funded under the Rashtriya Krishi Vikas Yojana (RKVY09VMC).

\section{References}

1. Baldwin CL, Goenka R (2006) Host immune responses to the intracellular bacterium Brucella: does the bacterium instruct the host to facilitate chronic infection? Crit Rev Immunol 26: 407-442. [Crossref]

2. Bellaire BH, Roop RM, Cardelli JA (2005) Opsonized virulent Brucella abortus replicates within nonacidic, endoplasmic reticulum-negative, LAMP-1-positive phagosomes in human monocytes. Infect Immun 73: 3702-1373. [Crossref]

3. Godfroid J, Garin-Bastuji B, Saegerman C, Blasco JM (2013) Brucellosis in terrestrial wildlife. Rev Sci Tech 32: 27-42. [Crossref]

4. Gupta V, Saxena HM (2017) A new bacteriophage-based luminescence assay for diagnosis of Brucellosis. Ind J exp biol 55: 296-302.

5. Gupta V, Saxena HM (2017) Isolation and characterization of BpL1, a broad acting lytic bacteriophage against Brucella. Int $J$ curr microbiol app sci 6: 2486-2496. [Crossref]
6. Jain L, Rawat M, Prajapati A, Tiwari AK, Kumar B, Chaturvedi VK (2015) Protective immune response of aluminium hydroxide gel adjuvanted phage lysate of Brucella abortus S19 in mice against direct virulent challenge with B abortus 544. Biologicals 43: 369-376. [Crossref]

7. Kollannur JD, Rathore R, Chauhan RS (2007) Epidemiology and economics of Brucellosis in animals and its zoonotic significance. Proceedings of XIII International Congress in Animal Hygiene. International Society for Animal Hygiene 466-468.

8. Morgan WJ, Mackinnon DT, Gill KPW, Gower SGM, Norris PIW (1978) Brucellosis Diagnosis: Standard Laboratory Techniques Report Series No. 1. MAFF, Weybridge, England.

9. OIE (2009) Bovine Brucellosis Section 2,4,3. Manual of the Diagnostic Tests and Vaccines for Terrestrial animals, vol 1, 5th Edn. Office International des Epizooties, Paris, France. 624-659.

10. Oliveira SC, Splitter GA (1994) Subcloning and expression of Brucella abortus L7 L12 ribosomal gene and T lymphocyte recognition of the recombinant protein. Infect Immun 62: 5201-5204.

11. Sawada T, Rimler RB, Rhoades KR (1982) Indirect Haemagglutination test that uses glutaraldehyde fixed sheep erythrocytes sensitized with exact antigens for detection of Pasturella antibody. J Clin Microbiol 15: 752-756.

12. Williams JE, Whitemore AD (1971) Serological diagnosis of Pullorum disease with the microagglutination system. Appl Microbiol 21: 392-399.

Copyright: (C2019 Raj S. This is an open-access article distributed under the terms of the Creative Commons Attribution License, which permits unrestricted use, distribution, and reproduction in any medium, provided the original author and source are credited. 